\title{
Novel NR4A1 Arg293Ser Mutation in Patients With Familial Crohn's Disease
}

\author{
KATSUHIRO MASAGO ${ }^{1,2}$ and SHIRO FUJITA ${ }^{1,2}$ \\ ${ }^{1}$ Department of Pathology and Molecular Diagnostics, Aichi Cancer Center, Aichi, Japan; \\ ${ }^{2}$ Division of Integrated Oncology, Institute of Biomedical Research and Innovation, Kobe, Japan
}

\begin{abstract}
Background/Aim: The underlying etiology of Crohn's disease remains unknown. The aim of this study was to identify genomic alterations associated with the development of Crohn's disease in one Japanese family with a family history of Crohn's disease. Materials and Methods: We performed whole-exome sequence and pedigree analysis of a Japanese family in which both sisters developed Crohn's disease. Whole-exome sequencing was performed using the Ion Torrent Proton ${ }^{T M}$ system. Data from the Proton runs were initially processed using the Ion Torrent platformspecific pipeline software Ion Reporter. An autosomal dominant mode of inheritance was assumed, and stringent selection criteria were applied. Results: A substitution in the NR4A1 gene at codon 293 resulting in an amino acid change from arginine to serine was identified only in the affected sisters. Conclusion: The impaired DNA-binding capacity of the NR4Al protein due to an NR4Al germline mutation may be a possible cause of Crohn's disease.
\end{abstract}

Crohn's disease (CD) (OMIM 266600) is an inflammatory bowel disease (IBD) that forms discontinuous erosions and ulcers throughout the digestive tract. Various genetic and environmental factors (1) and several risk loci associated with susceptibility to $\mathrm{CD}$ have been reported $(2,3)$. However, the definitive etiology remains elusive.

In this study, we used whole-exome sequencing and pedigree analysis of a Japanese family with $\mathrm{CD}$ to evaluate which germline alterations affected the onset of $\mathrm{CD}$.

This article is freely accessible online.

Correspondence to: Katsuhiro Masago, Department of Pathology and Molecular Diagnostics, Aichi Cancer Center, 1-1 Kanokoden, Chikusa-ku, Nagoya City, Aichi 464-8681 Japan. Tel: +81 527626111, Fax: +81 527649897, e-mail: masago@aichi-cc.jp

Key Words: Crohn's disease, exome sequence, germline mutation, Nur77, steroid orphan receptor.

\section{Materials and Methods}

Written informed consent for this study and publication were obtained from all study participants and their parents. The study was approved by the Research Ethics Committee of the Institute of Biomedical Research and Innovation (No. 16-02) and conducted in accordance with the ethical principles of the Declaration of Helsinki.

Patients and family members. Patients III-6 and III-7 were diagnosed with CD at the ages of 18 and 20, respectively. Their initial symptoms were abdominal pain, diarrhea, and bloody stool. Their unaffected father (II-3) has a history of sarcoidosis at the age of 27. He was diagnosed with a chest computed tomography (CT) and his condition had regressed without treatment.

Mutation detection: Whole-exome sequencing. Whole-exome sequencing was performed using the Ion Torrent Proton ${ }^{\mathrm{TM}}$ system (Thermo Fisher Scientific, Waltham, MA, USA) in conjunction with the Ion AmpliSeq ${ }^{\mathrm{TM}}$ Exome RDY Kit PIV3 (Thermo Fisher Scientific).

Ion Torrent library preparation and sequencing. An Ion Torrent adapter-ligated library was generated following the Ion AmpliSeq ${ }^{\mathrm{TM}}$ Exome RDY Kit Library Preparation manufacturer's protocol (Thermo Fisher Scientific, MAN0010084). Briefly, 100 ng of gDNA was used as the starting material in the AmpliSeq exome amplification step. The amplicons were end-repaired, and the Ion Torrent P1 adapters and amplicons were ligated using DNA ligase. Barcoding was performed using the Ion DNA Barcoding kit (Thermo Fisher Scientific). Following AMPure bead purification (Beckman Coulter, CA, USA), the concentrations and sizes of the library components were determined using the Life Technologies StepOne ${ }^{\mathrm{TM}}$ system (Thermo Fisher Scientific) and Ion Library TaqMan ${ }^{\circledR}$ Quantitation Assay Kit (Thermo Fisher Scientific). Sample emulsion PCR, emulsion breaking, and enrichment were performed using the Ion PI ${ }^{\mathrm{TM}} \mathrm{Hi}-\mathrm{Q}$ Chef Kit (Thermo Fisher Scientific) according to the manufacturer's instructions. An input concentration of one DNA template copy/ion sphere particle (ISP) was added to the emulsion PCR master mix, and the emulsion was generated using the Ion Chef $^{\mathrm{TM}}$ system (Thermo Fisher Scientific). Ion PI chips (version 3) and the Ion Torrent Proton system were used to enrich templatepositive ISPs and to perform the sequencing.

Variant calling. Data from the Proton runs were initially processed using the Ion Torrent platform-specific pipeline software Ion Reporter (version 5.0) to generate sequence reads, trim adapter 
Table I. Filtering steps for homozygous variants.

\begin{tabular}{ll}
\hline Filtering criteria for SNVs and indels & $\begin{array}{l}\text { Number of missense, } \\
\text { nonsense or stop-loss } \\
\text { mutations after filtering }\end{array}$
\end{tabular}

Coding exons shared by II-1, III-1 and III-2

Protein expression according

19

to the Human Protein Atlas

SIFT score less than 0.05 or not available

PolyPhen-2 score 0.85 to 1.0

Result obtained by FATHMM (<-2.0)

Result obtained by CADD score $(>30)$

SNVs/indels $\leq 1 \%$ in 5000 Exomes database

and 1000 Genome database SNVs/indels not

in eitherdbSNP 138 or UCSC Common SNPs

SNVs/indels $\leq 0.1 \%$ in ToMMo database (2KJPN)

Confirmed by Sanger sequencing
14

14

14

8

2

2

2

2
CADD: Combined annotation dependent depletion; FATHMM: functional analysis through Hidden Markov Models (v2.3); PolyPhen: polymorphism phenotyping; SIFT: sorting intolerant from tolerant; ToMMo: Tohoku Medical Megabank Organization.

sequences, and filter and to remove poor signal-profile reads using the optimized parameters provided by the manufacturer of the AmpliSeq exome. The other filter employed was visual examination of the mutations using CLC Genomics Workbench version 9.0.1 (Qiagen, Hilden, Germany) in addition to the removal of possible strand-specific errors (i.e., a mutation detected only in either the "plus" or "minus" strand but not in both strands). A limitation of this analysis is that structural variants such as large deletions or exon skipping variants could not be detected.

\section{Results}

Whole-exome sequencing was performed with DNA samples from patients III-6 and III-7, their unaffected father (II-3), and their unaffected mother (II-4) (Figure 1A). A mutation at codon 293 resulting in an amino acid substitution from arginine to serine, Arg293Ser, was identified in NR4Al in patient III-6, patient III-7, and their unaffected father (II-3) (Figure 1B). An autosomal dominant mode of inheritance, which is speculative, was assumed, and stringent selection criteria were applied (Tables I and II). The variant did not exist in the data of 10 non-consanguineous, unrelated persons whose library was created using AmpliSeq Exome Kit and sequenced by Ion Torrent System as in the present study.

\section{Discussion}

NR4A1 was initially known as a nerve growth factor (4), and was also identified as a member of the "orphan" nuclear steroid receptor superfamily (5). NR4A1 is transcriptionally active in many types of cells $(6,7)$ under different culture conditions. Furthermore, the suppression of NR4A1 can
Table II. Characteristics of the variants identified in the affected members of the studied family.

\begin{tabular}{lcc}
\hline & NR4A1 & ICK \\
\hline Genomic position (hg19) & chr12: 52449814 & chr6:52906023 \\
DNA variant & c.877C $>$ T & c.11_12insGATA \\
Protein alteration & p.Arg293Ser & p.Tyr4* \\
Predicted effect of & & \\
missense variants & 0: probably damaging & N/A \\
SIFT & 1: probably damaging & N/A \\
PolyPhen-2 & $-5.32:$ deleterious & N/A \\
FATHMM & 35: pathogenic & 35: pathogenic \\
CADD score & -1.16 Kcal/mol: & N/A \\
mCSM-NA* $(\Delta \Delta G)$ & reduced DNA & \\
& binding affinity & \\
Evolutionary conservation & & 2.55 \\
PhyloP & 2.55
\end{tabular}

CADD: Combined annotation dependent depletion; ICK: intestinal cell kinase; FATHMM: functional analysis through Hidden Markov Models (v2.3); mCSM-NA: effects of mutations on protein-nucleic acids interactions; N/A: not applicable; PhyloP: phylogenetic $p$-values; PolyPhen: polymorphism phenotyping; SIFT: sorting intolerant from tolerant. *http://biosig.unimelb.edu.au/mcsm_na/prediction.

inhibit apoptosis (8). Moreover, NR4A1 plays a significant role in the functioning of macrophages (9). The loss of NR4A1 in myeloid cells leads to the acceleration of leukocyte infiltration to the central nervous system (10). The loss of NR4A1 also exacerbates organ fibrosis in the experimental mouse model by dysregulating transforming growth factor- $\beta$ pathway (11). Thus, NR4A1 is involved in the regulation of fundamental cellular functions, including inflammation and cell survival $(12,13)$.

The NR4A1 gene contains seven exons. Exon2 encodes an $\mathrm{N}$-terminal transactivation domain, exon 3 and 4 encode a DNA-binding domain, and dimerization and ligand-binding domains are encoded by exons 5 to 7 (14). The DNA-binding domain is a highly conserved amino acid sequence also found in other DNA-binding proteins such as the retinoid $\mathrm{X}$ receptor (RXR), estrogen receptor 2, steroid receptor, and androgen receptor (Figure 2B). This cysteine-rich region appears to fold into two zinc fingers. The DNA-binding domain interacts with cis-acting elements of the regulated genes (15). In the case of the androgen receptor, a mutation within the DNAbinding domain (Arg585Lys) is linked to a positive receptorbinding form that exhibits androgen insensitivity (16). The identified Arg293Ser variant (Figure 2A and B), which is equivalent to Arg585Lys identified in androgen insensitivity syndromes, is located in the DNA-binding domain in exon 4 (Figure 2B) and results in a severely impaired DNA-binding capacity. This Arg293Ser mutation is extremely rare in the general population, and was not documented in the Database for Genome Aggregation. 


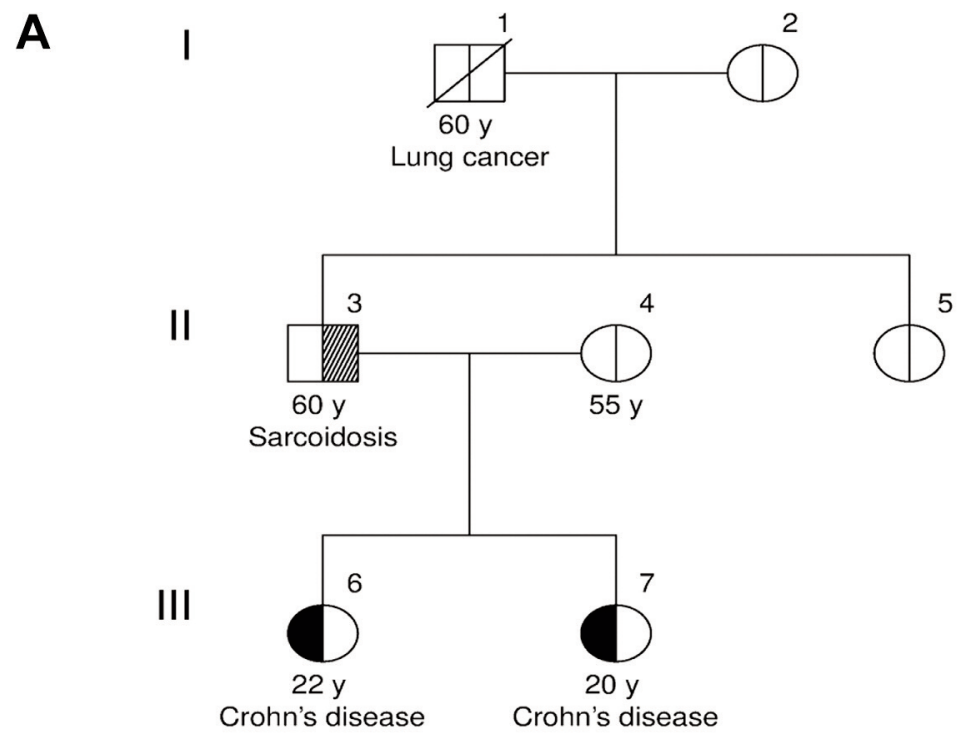

B

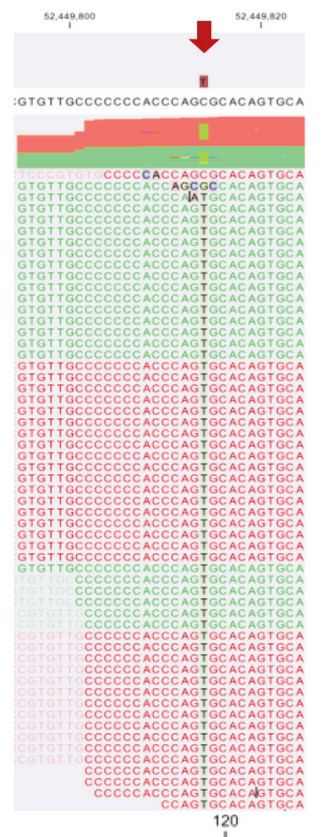

ACCCAGTGCACAGTGCA

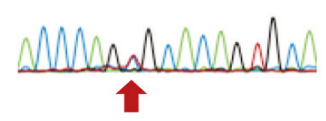

II-1:
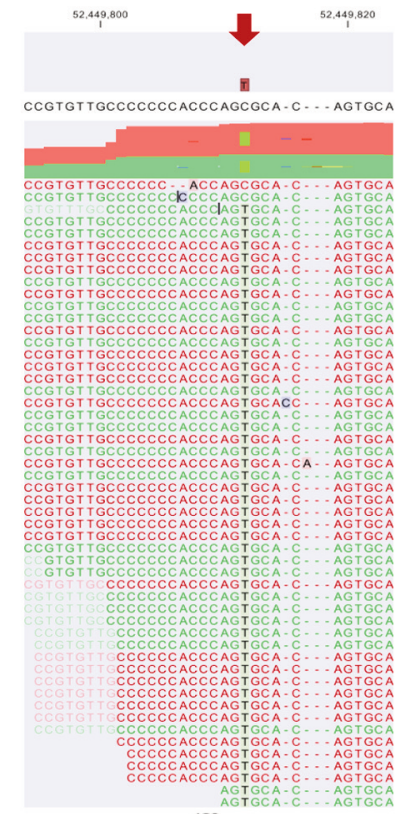

120

ACCCAGTGCACAGTGCA

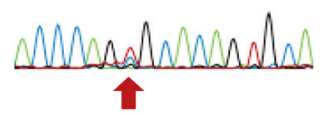

III-1:

Patient

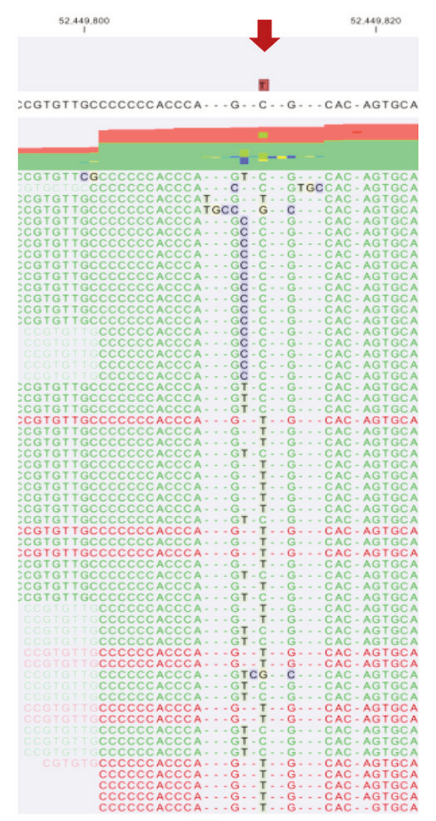

120

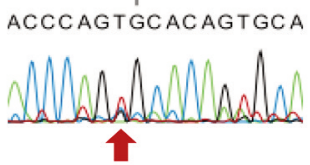

III-2:

Patient

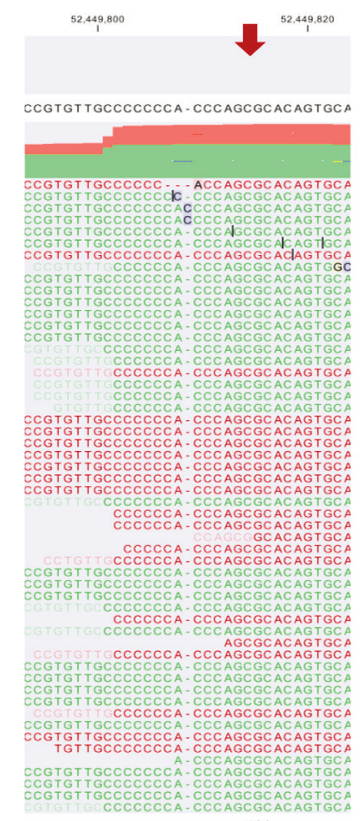

120

ACCCAGCGCACAGTGCA

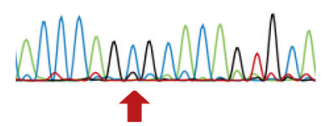

II-3:

A1

A2

Figure 1. Pedigree plotting and sequencing results. A). Pedigree of the study family and the disease status of each member. Squares denote male family members, circles denote female members, shaded symbols denote affected members, and slashes denote deceased members. We performed whole-exome sequencing using DNA from peripheral blood samples from the following four family members: II-1, II-3, III-1, and III-2. B). Sequence chromatogram and direct sequence chart of the mutant genome. The NR4A1 germline mutation $c .877 C>T$, a missense substitution of cysteine for arginine at position 293 (encoding p.Arg293Cys; position chr12:52449814 T/C), was detected with Ion Torrent technology. 


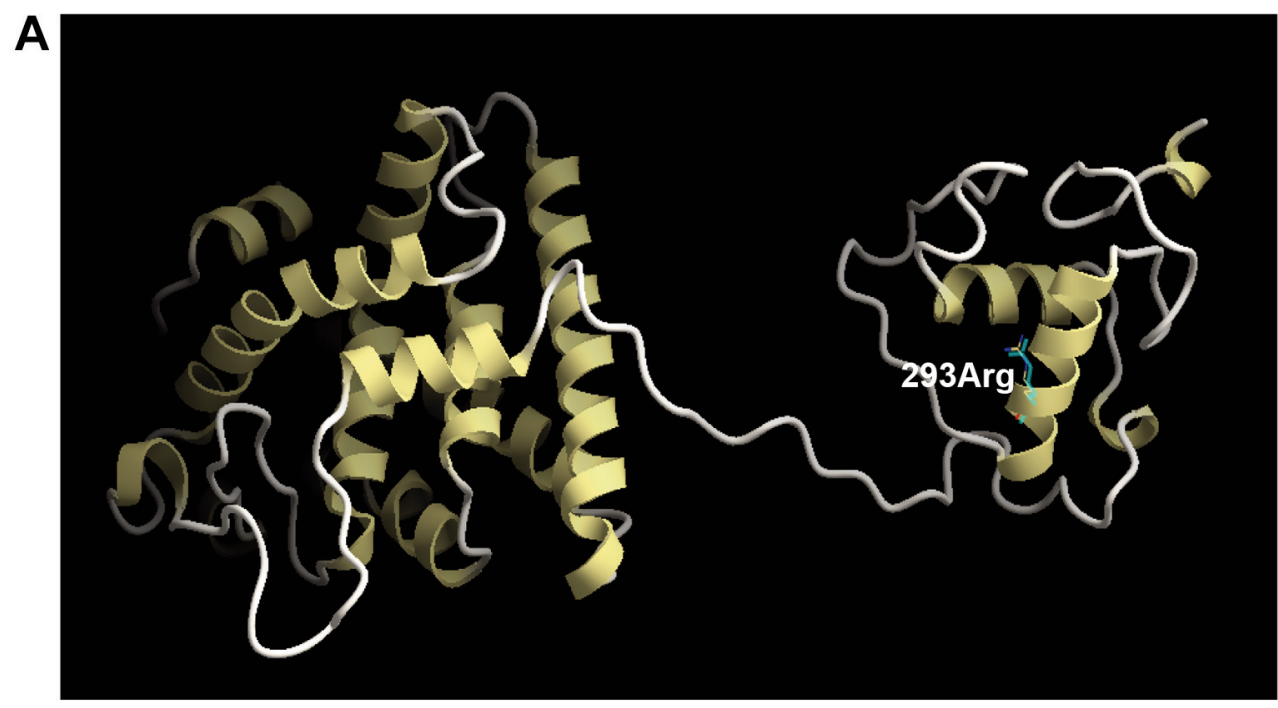

B

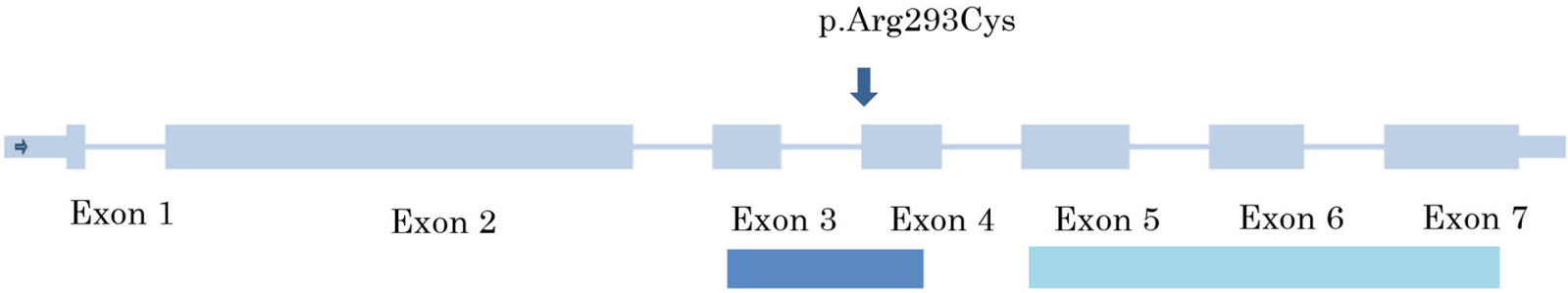

DNA-binding domain (264-339)

Ligand-binding domain (360-595)

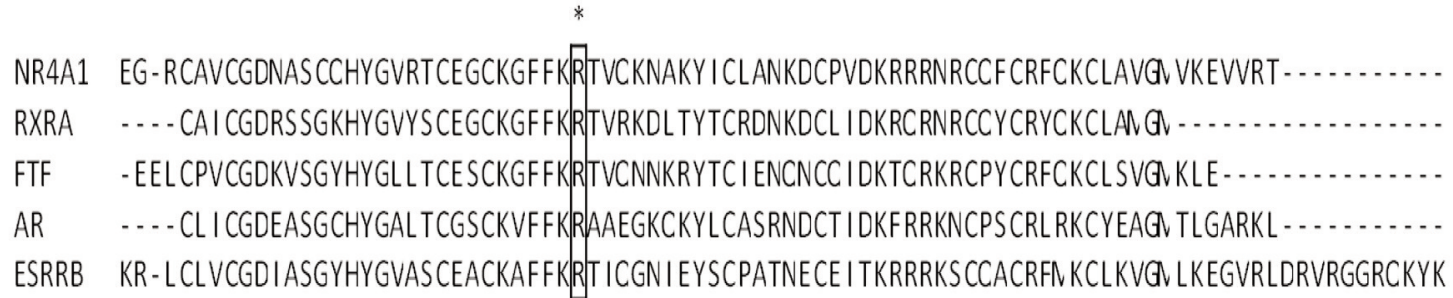

Figure 2. Structure of mutated NR4A1. A). Crystal structure of the DNA-binding domain of the Arg293-containing protein. B). DNA-binding domain structures of human proteins and the conservation of the Cys293 position across human proteins. The cysteine at this position in the strand is responsible for binding to the promoter region of target genes.

There have been two reports on experimental colitis using the $N R 4 A 1^{-1-}$ mouse model. In the first report, Hamers et al. defined the role of NR4A1 in IBD in induced mouse IBD models. Inflammation was increased in $N R 4 A 1^{-1-}$ mice and the mediated invasion of T-cells and monocytes into the colon was more pronounced. The expression of MCP-1, TNF $\alpha$ and IL- 6 was increased and the transcription of Foxp3 was decreased. Moreover, the over-expression of NR4A1 in activated RAW macrophages contributed to the upregulation of IL-10 and the down-regulation of TNF $\alpha$, MIF-1, and MCP-1 transcription via NFkB repression. The authors concluded that NR4A1 has a protective function in the induced colitis model (17).

In the second report, $\mathrm{Wu}$ et al. found genetic variants of NR4Al associated with susceptibility to both ulcerative colitis (UC) and CD in the most recent GWASs on UC and CD. They confirmed that NR4A1 expression was suppressed in the colon tissues of UC or CD patients. They also revealed that deficiency 
of NR4A1 increased vulnerability to colitis, whereas treatment with an NR4A1 agonist significantly reduced the induced colitis in the $N R 4 A I^{-1-}$ mouse model. The authors concluded that NR4A1 was a key regulator of the TRAF6/TLR/IL-1R-initiated inflammatory pathway in IBD (18).

In an in vitro model, $\mathrm{Nr} 4 \mathrm{al}$ deletion in $\mathrm{B} 2$ cells increased atherosclerosis and was associated with increased $\mathrm{T}$ follicular helper cell-Germinal Center response and NR4A1 expression in Marginal Zone B cells regulating PD-L1 expression, limiting the $\mathrm{T}$ follicular helper cell-Germinal Center response and protecting from atherosclerosis (19). The NR4AI expression was elevated in human osteoarthritis cartilage and in an in vitro osteoarthritis model, which could be blocked by the NF-kB signal inhibitor JSH23 (20).

Recently, Klepsh et al. identified that the loss of NR2F6 induced spontaneous late-onset colitis in the $N R 2 \mathrm{Fr}^{-1-}$ mouse model (21). They suggested that agonists of NR2F6 might be a potential treatment strategy of human IBD. Onuki et al. reported that the activation of $\mathrm{RXR} /$ peroxisome proliferator-activated receptor $\delta$ and NR4A1/RXR heterodimers diminished the monocyte-mediated inflammatory response in the gut (22). Liu et al. also reported that NR4A1 is an important regulator of T cell function and a potential target for tumor immunotherapy (23). These observations also support our findings.

In conclusion, we speculate that the onset of $\mathrm{CD}$ in this family may be due to the impaired DNA-binding capacity of the NR4A1 protein caused by the NR4Al Arg293Ser germline mutation. As treatment with an agonist for NR4A1 has been reported to attenuate excessive inflammation in an experimental mouse colitis model (18), NR4A1 protein impairment may be a possible cause of IBD. NR4A1 agonists may be future targets for preventing and treating IBD, and screening for NR4AI germline mutations will provide important information that can help elucidate the etiology of IBD in the future.

\section{Conflicts of Interest}

The Authors have no conflicts of interest to declare regarding this study.

\section{Authors' Contributions}

KM: project development, manuscript writing, study conceptualization and design, data collection and abstraction, and statistical analyses; SF: study conceptualization and design, and manuscript preparation and revision; All Authors read and approved the final version of the manuscript.

\section{References}

1 Xavier R and Podolsky D: Unravelling the pathogenesis of inflammatory bowel disease. Nature 448(7152): 427-434, 2020. PMID: 17653185. DOI: 10.1038/nature06005
2 Momozawa Y, Dmitrieva J, Théâtre E, Deffontaine V, Rahmouni S, Charloteaux B, Crins F, Docampo E, Elansary M, Gori AS, Lecut C, Mariman R, Mni M, Oury C, Altukhov I, Alexeev D, Aulchenko Y, Amininejad L, Bouma G, Hoentjen F, Löwenberg M, Oldenburg B, Pierik MJ, Vander Meulen-de Jong AE, Janneke van der Woude C, Visschedijk MC, International IBD Genetics Consortium., Lathrop M, Hugot JP, Weersma RK, De Vos M, Franchimont D, Vermeire S, Kubo M, Louis E and Georges M: IBD risk loci are enriched in multigenic regulatory modules encompassing putative causative genes. Nat Commun 9(1): 2427, 2018. PMID: 29930244. DOI: 10.1038/s41467-018-04365-8

3 Alonso A, Domènech E, Julià A, Panés J, García-sánchez V, Mateu P, Gutiérrez A, Gomollón F, Mendoza J, Garcia-planella E, Barreiro-de acosta M, Muñoz F, Vera M, Saro C, Esteve M, Andreu M, Chaparro M, Manyé J, Cabré E, López-lasanta M, Tortosa R, Gelpí J, García-montero A, Bertranpetit J, Absher D, Myers R, Marsal S and Gisbert J: Identification of risk loci for Crohn's disease phenotypes using a genome-wide association study. Gastroenterology 148(4): 794-805, 2021. PMID: 25557950. DOI: 10.1053 /j.gastro.2014.12.030

4 Milbrandt J: Nerve growth factor induces a gene homologous to the glucocorticoid receptor gene. Neuron 1(3): 183-188, 2020. DOI: 10.1016/0896-6273(88)90138-9

5 Safe S, Jin U, Morpurgo B, Abudayyeh A, Singh M and Tjalkens R: Nuclear receptor 4A (NR4A) family - orphans no more. J Steroid Biochem Mol Biol 157: 48-60, 2020. PMID: 25917081. DOI: $10.1016 /$ j.jsbmb.2015.04.016

6 Davis I, Hazel T, Chen R, Blenis J and Lau L: Functional domains and phosphorylation of the orphan receptor Nur77. Mol Endocrinol 7(8): 953-964, 2017. PMID: 8232315. DOI: 10.1210/mend.7.8.8232315

7 Paulsen RE, Weaver CA, Fahrner TJ and Milbrandt J: Domains regulating transcriptional activity of the inducible orphan receptor NGFI-B. J Biol Chem 267(23): 16491-16496, 1992. PMID: 1644831.

8 Woronicz J, Calnan B, Ngo V and Winoto A: Requirement for the orphan steroid receptor Nur77 in apoptosis of T-cell hybridomas. Nature 367(6460): 277-281, 2020. PMID: 8121493. DOI: $10.1038 / 367277 \mathrm{a} 0$

9 Pei L, Castrillo A and Tontonoz P: Regulation of macrophage inflammatory gene expression by the orphan nuclear receptor Nur77. Molecular Endocrinology 20(4): 786-794, 2020. PMID: 16339277. DOI: $10.1210 /$ me.2005-0331

10 Shaked I, Hanna R, Shaked H, Chodaczek G, Nowyhed H, Tweet G, Tacke R, Basat A, Mikulski Z, Togher S, Miller J, Blatchley A, Salek-ardakani S, Darvas M, Kaikkonen M, Thomas G, Lai-wing-sun S, Rezk A, Bar-or A, Glass C, Bandukwala $\mathrm{H}$ and Hedrick $\mathrm{C}$ : Transcription factor Nr4a1 couples sympathetic and inflammatory cues in CNS-recruited macrophages to limit neuroinflammation. Nat Immunol 16(12): 1228-1234, 2020. PMID: 26523867. DOI: 10.1038/ni.3321

11 Palumbo-zerr K, Zerr P, Distler A, Fliehr J, Mancuso R, Huang J, Mielenz D, Tomcik M, Fürnrohr B, Scholtysek C, Dees C, Beyer C, Krönke G, Metzger D, Distler O, Schett G and Distler $\mathrm{J}$ : Orphan nuclear receptor NR4A1 regulates transforming growth factor- $\beta$ signaling and fibrosis. Nature Medicine 21(2): 150-158, 2019. PMID: 25581517. DOI: 10.1038/nm.3777

12 Pols T, Bonta P and De vries C: NR4A nuclear orphan receptors: protective in vascular disease? Curr Opin Lipidol 18(5): 515-520, 2021. PMID: 17885421. DOI: 10.1097/MOL.0b013e3282ef77d1 
13 Maxwell M and Muscat G: The NR4A Subgroup: Immediate early response genes with pleiotropic physiological roles. Nucl Recept Signal 4(1): nrs.04002, 2020. PMID: 16604165. DOI: $10.1621 / \mathrm{nrs} .04002$

14 Saucedo-cardenas O, Kardon R, Ediger T, Lydon J and Conneely $\mathrm{O}$ : Cloning and structural organization of the gene encoding the murine nuclear receptor transcription factor, NURR1. Gene 187(1): 135-139, 2020. PMID: 9073077. DOI: 10.1016/s03781119(96)00736-6

15 Ham J, Thomson A, Needham M, Webb P and Parker M: Characterization of response elements for androgens, glucocorticoids and progestins in mouse mammary tumour virus. Nucleic Acids Res 16(12): 5263-5276, 2017. PMID: 2838812. DOI: $10.1093 / \mathrm{nar} / 16.12 .5263$

16 Sultan C, Lumbroso S, Poujol N, Belon C, Boudon C and Lobaccaro J: Mutations of androgen receptor gene in androgen insensitivity syndromes. J Steroid Biochem Mol Biol 46(5): 519530, 2019. PMID: 8240973. DOI: 10.1016/0960-0760(93)90178-y

17 Hamers A, Van dam L, Teixeira duarte J, Vos M, Marinković G, Van tiel C, Meijer S, Van stalborch A, Huveneers S, Te velde A, De jonge $\mathrm{W}$ and De vries C: Deficiency of nuclear receptor Nur77 aggravates mouse experimental colitis by increased NFKB activity in macrophages. PLOS ONE 10(8): e0133598, 2020. PMID: 26241646. DOI: 10.1371/journal.pone.0133598

18 Wu H, Li X, Wang J, Gan W, Jiang F, Liu Y, Zhang X, He X, Zhao Y, Lu X, Guo Y, Zhang X and Li J: NUR77 exerts a protective effect against inflammatory bowel disease by negatively regulating the TRAF6/TLR-IL-1R signalling axis. J Pathol 238(3): 457-469, 2019. PMID: 26564988. DOI: 10.1002/ path. 4670

19 Nus M, Basatemur G, Galan M, Cros-brunsó L, Zhao T, Masters L, Harrison J, Figg N, Tsiantoulas D, Geissmann F, Binder C, Sage A and Mallat Z: NR4A1 Deletion in Marginal Zone B Cells Exacerbates Atherosclerosis in Mice-Brief Report. Arterioscler Thromb Vasc Biol 40(11): 2598-2604, 2020. PMID: 32907369. DOI: 10.1161/atvbaha.120.314607
20 Xiong Y, Ran J, Xu L, Tong Z, Adel abdo M, Ma C, Xu K, He $\mathrm{Y}, \mathrm{Wu} \mathrm{Z}$, Chen Z, Hu P, Jiang L, Bao J, Chen W and Wu L: Reactivation of NR4A1 Restrains Chondrocyte Inflammation and Ameliorates Osteoarthritis in Rats. Front Cell Dev Biol 8: 158, 2020. PMID: 32258036. DOI: 10.3389/fcell.2020.00158

21 Klepsch V, Gerner R, Klepsch S, Olson W, Tilg H, Moschen A, Baier G and Hermann-kleiter N: Nuclear orphan receptor NR2F6 as a safeguard against experimental murine colitis. Gut 67(8): 1434-1444, 2020. PMID: 28779026. DOI: 10.1136/gutjnl-2016313466

22 Onuki M, Watanabe M, Ishihara N, Suzuki K, Takizawa K, Hirota M, Yamada T, Egawa A, Shibahara O, Nishii M, Fujihara M, Makishima M, Takahashi D, Furusawa Y, Kakuta H and Hase $\mathrm{K}$ : A partial agonist for retinoid $\mathrm{X}$ receptor mitigates experimental colitis. Int Immunol 31(4): 251-262, 2019. PMID: 30590577. DOI: 10.1093/intimm/dxy089

23 Chen J, López-moyado I, Seo H, Lio C, Hempleman L, Sekiya T, Yoshimura A, Scott-browne J and Rao A: NR4A transcription factors limit CAR $\mathrm{T}$ cell function in solid tumours. Nature 567(7749): 530-534, 2020. PMID: 30814732. DOI: 10.1038/ s41586-019-0985-x 